\title{
RESOLUTIONS OF 2 AND 3 DIMENSIONAL RINGS OF INVARIANTS FOR CYCLIC GROUPS
}

\author{
JOHN C. HARRIS AND DAVID L. WEHLAU
}

\begin{abstract}
Let $G$ be the cyclic group of order $n$ and suppose $\mathbf{F}$ is a field containing a primitive $n^{\text {th }}$ root of unity. We consider the ring of invariants $\mathbf{F}[W]^{G}$ of a three dimensional representation $W$ of $G$ where $G \subset \mathrm{SL}(W)$. We describe minimal generators and relations for this ring and prove that the lead terms of the relations are quadratic. These minimal generators for the relations form a Gröbner basis with a surprisingly simple combinatorial structure. We describe the graded Betti numbers for a minimal free resolution of $F[W]^{G}$. The case where $W$ is any two dimensional representation of $G$ is also handled.
\end{abstract}

\section{INTRODUCTION}

Let $n \geq 3$ be a positive integer and let $G$ denote the cyclic group of order $n$. Let $\mathbf{F}$ be a field which contains a primitive $n^{\text {th }}$ root of unity, $\epsilon$. Let $\sigma$ denote a fixed generator of $G$. There are $n$ inequivalent irreducible representations of $G$ over $\mathbf{F}$, each of dimension 1 . We denote these by $W_{b}$ with $b=1,2, \ldots, n$ where $G$ acts on $W_{b}$ via $\sigma \cdot w=\epsilon^{-b} w$ for all $w \in W_{b}$.

In this paper, we compute the graded Betti numbers for the minimal graded free resolutions for the invariant rings $\mathbf{F}[W]^{G}$, when $W=W_{b} \oplus$ $W_{c}$ is two dimensional or $W=W_{b} \oplus W_{c} \oplus W_{d}$ is three dimensional and $G \subset \mathrm{SL}(W)$. In each case, we first describe a minimal set of generators for $\mathbf{F}[W]^{G}$ (taken to be monomials since the $G$ action is diagonal) to produce a short exact sequence:

$$
0 \rightarrow J \rightarrow S=\mathbf{F}\left[x_{1}, \ldots, x_{m}\right] \stackrel{\pi}{\longrightarrow} \mathbf{F}[W]^{G} \rightarrow 0 .
$$

By a careful choice of monomial ordering on the ring $S$, we describe a minimal set of homogeneous generators $\left\{R_{i, j}\right\}$ (with $\operatorname{LT}\left(R_{i, j}\right)=x_{i} x_{j}$ ) for $J$ that is also a Gröbner basis for $J$. In Section 5 we find the graded Betti numbers for the monomial ideals $\operatorname{LT}(J)$ in $S$. It turns out that

Date: November 10, 2018.

2010 Mathematics Subject Classification. 13A50; 13D02.

Key words and phrases. invariant theory, minimal resolutions, Betti numbers, monomial ideals, Gröbner bases, cyclic group. 
these minimal graded free resolutions are pure (the differentials have a fixed polynomial degree), so that the graded Betti numbers for $\operatorname{LT}(J)$ and for $J$ are the same.

The two and three dimensional cases are treated in Sections 3 and 4 , respectively. In the three dimensional case, we have $W=W_{b} \oplus W_{c} \oplus W_{d}$, $0<b, c, d<n, G \subset \mathrm{SL}(W)$, and $\mathbf{F}[W]=\mathbf{F}[x, y, z]$. Since $G \subset \mathrm{SL}(W)$ we have that $n$ divides $b+c+d$, so that the product of the three linear variables $a=x y z$ is a minimal (since $0<b, c, d<n$ ) generator of $\mathbf{F}[W]^{G}$. It follows that all other minimal generators involve only two of the variables and so correspond to generators arising in the two dimensional case. We will describe a set of $m+1$ minimal generators for this ring to produce a short exact sequence:

$$
0 \rightarrow J \rightarrow S=\mathbf{F}\left[A, B_{1}, B_{2}, \ldots, B_{m}\right] \stackrel{\pi}{\longrightarrow} \mathbf{F}[W]^{G} \rightarrow 0
$$

Also we will describe elements $R_{i, j}$ for certain $i, j$ with $\operatorname{LT}\left(R_{i, j}\right)=B_{i} B_{j}$, that form both a minimal generating set for $J$ and also a Gröbner basis for $J$. In the paper [CHW], an explicit minimal resolution was constructed for this lead term ideal $\operatorname{LT}(J)$ over $S$.

We would like to thank the referee for a number of expository suggestions and for a simplified proof of Proposition 5.3.

\section{Minimal Resolutions and Graded Betti Numbers}

In this section we describe minimal resolutions and some related ideas. We refer the reader to the books [CLO] and [M-S] for further details.

Let $R=\mathbf{F}\left[x_{1}, x_{2}, \ldots, x_{m}\right]$ be a graded polynomial ring with each $\operatorname{deg}\left(x_{i}\right)$ a positive integer, and let $I$ be a homogeneous ideal in $R$. Then the minimal graded free resolution of $R / I$ has the form:

$$
0 \rightarrow M_{k} \rightarrow M_{k-1} \rightarrow \cdots \rightarrow M_{1} \rightarrow M_{0}=R \rightarrow R / I \rightarrow 0
$$

where $k \leq m, M_{i}=\bigoplus_{j} R(-j)^{\beta_{i, j}(R / I)}$, and $R(-j)$ is the free $R$-module obtained by shifting the degrees of $R$ by $j$. The number $\beta_{i, j}(R / I)$, the $(i, j)$-th graded Betti number of $R / I$, equals the number of minimal generators of degree $j$ in the $i$-th syzygy module of $R / I$.

One also defines graded Betti numbers, $\beta_{i, j}(I)$, for the ideal $I$ as follows: if $\cdots \rightarrow M_{1} \rightarrow M_{0} \rightarrow R / I \rightarrow 0$ is a minimal graded free resolution of $R / I$, then $\cdots \rightarrow M_{1} \rightarrow I \rightarrow 0$ is a minimal graded free resolution of $I$. Using the standard conventions that $\beta_{-1,0}(I)=1$ and $\beta_{-1, j}(I)=0$ for all $j>0$, it follows that $\beta_{i-1, j}(I)=\beta_{i, j}(R / I)$ for all $i, j \geq 0$.

If we are given a monomial order for $R=\mathbf{F}\left[x_{1}, \ldots, x_{m}\right]$ and if $I$ is a homogeneous ideal in $R$, then we let $\operatorname{LT}(I)$ be the ideal generated by 
the leading terms of the elements of $I$. A Gröbner basis for $I$ is a set of elements of $I$ whose lead terms generate LT $(I)$. By a simple lead term argument it can be shown that the elements of a Gröbner basis for $I$ generate $I$. Since $\operatorname{LT}(I)$ is a monomial ideal, it makes sense to consider the polynomial degree of elements (defined by setting the degrees of the $x_{i}$ equal to 1.) So for $\mathrm{LT}(I)$ we have two sets of Betti numbers, which we will call the graded Betti numbers and the polynomial Betti numbers.

In general, the minimal graded free resolutions of $R / I$ and $R / \mathrm{LT}(I)$ can have different graded Betti numbers. However, in some cases, they are equal.

Lemma 2.1. ([CHW] Section 6) Let I be a homogeneous ideal in the graded ring $R=\mathbf{F}\left[x_{1}, \ldots, x_{s}\right]$ minimally generated by a Gröbner basis, $\left\{z_{1}, \ldots, z_{k}\right\}$, where, for each $i$, the polynomial degree of the lead term of $z_{i}$ is less than or equal to the polynomial degree of the other terms of $z_{i}$. If $\left(M_{*}, d_{*}\right)$ is a minimal graded free resolution of $R / L T(I)$ that is pure (i.e., where each of the differentials is homogeneous with respect to the polynomial degree), then there exist differentials $\widehat{d}_{*}$ so that $\left(M_{*}, \widehat{d}_{*}\right)$ is a minimal graded free resolution of $R / I$. In particular, the graded Betti numbers for $R / I$ and $R / \mathrm{LT}(I)$ are the same.

In this paper, we are particularly interested in the graded Betti numbers for invariant rings $\mathbf{F}[W]^{G}$. These are defined as follows: if $\left\{b_{1}, \ldots, b_{m}\right\}$ is a minimal set of homogeneous generators for $\mathbf{F}[W]^{G}$, then there is a homomorphism from $R=\mathbf{F}\left[x_{1}, \ldots, x_{m}\right]$ onto $\mathbf{F}[W]^{G}$, where $\operatorname{deg}\left(x_{i}\right)=\operatorname{deg}\left(b_{i}\right)$ and $x_{i}$ maps to $b_{i}$. The kernel of this homo-

morphism, say $I$, is homogeneous and is called the ideal of relations for $\mathbf{F}[W]^{G}$. Since $\mathbf{F}[W]^{G} \cong R / I$ as graded rings, we let $\beta_{i, j}\left(\mathbf{F}[W]^{G}\right)=$ $\beta_{i, j}(R / I)$.

It will turn out that Lemma 2.1 applies to all of the invariant rings considered in this paper.

\section{Two Dimensional Case}

Suppose $W=W_{b} \oplus W_{c}$ is a 2 dimensional representation of $G$ with $0<b, c<n$. Then we have $\mathbf{F}[W]=\mathbf{F}[x, y]$ where $\sigma \cdot x=\epsilon^{b} x$ and $\sigma \cdot y=\epsilon^{c} y$. It is easy to see that there exist minimal generating sets for the invariant ring $\mathbf{F}[W]^{G}$ consisting of monomials. Let $\left\{u_{i}=\right.$ $\left.x^{a_{i}} y^{b_{i}} \mid i=1,2, \ldots, m\right\}$ be a set of minimal generators: $\mathbf{F}[W]^{G}=$ $\mathbf{F}\left[u_{1}, u_{2}, \ldots, u_{m}\right]$. Reordering, if necessary, we may assume that $a_{1}>$ $a_{2}>\cdots>a_{m-1}>a_{m}$. Since $m$ is minimal, $u_{i}$ does not divide $u_{j}$ for $i \neq j$. This implies that $b_{1}<b_{2}<\cdots<b_{m-1}<b_{m}$. Moreover, it is clear that $a_{1}=n / \operatorname{gcd}(n, b), b_{m}=n / \operatorname{gcd}(n, c)$ and $a_{m}=0=b_{1}$. 
Consider the short exact sequence

$$
0 \rightarrow J \rightarrow S=\mathbf{F}\left[U_{1}, U_{2}, \ldots, U_{m}\right] \stackrel{\pi}{\longrightarrow} \mathbf{F}[W]^{G} \rightarrow 0
$$

where the $U_{i}$ are indeterminants, $\pi\left(U_{i}\right)=u_{i}$ for $i=1,2, \ldots, m$, and $J$ is the ideal of relations for $\mathbf{F}[W]^{G}$. We put $\operatorname{deg}\left(U_{i}\right):=\operatorname{deg}\left(u_{i}\right)$ and work with the following monomial order on $S$, for monomials $\alpha=\prod_{i=1}^{m} U_{i}^{e_{i}}$ and $\beta=\prod_{i=1}^{m} U_{i}^{f_{i}}$, we say $\alpha<\beta$ if

(1) $\operatorname{deg}(\alpha)<\operatorname{deg}(\beta)$;

(2) $\operatorname{deg}(\alpha)=\operatorname{deg}(\beta)$ and $e_{i}<f_{i}$ for the largest $i$ where $e_{i} \neq f_{i}$.

For a homogeneous polynomial $f$ in $S$ : $\operatorname{LT}(f)$ and $\operatorname{LM}(f)$ will denote the lead term and lead monomial, respectively.

We now describe a minimal generating set for the ideal of relations $J$. For each pair $i, j$, with $1 \leq i, j \leq m$ and $j-i \geq 2$, consider the product $u_{i} u_{j} \in \mathbf{F}[W]^{G}$. The monomial $u_{i+1}$ properly divides $u_{i} u_{j}$ and so $\alpha:=\left(u_{i} u_{j}\right) / u_{i+1}=x^{a_{i}+a_{j}-a_{i+1}} y^{b_{i}+b_{j}-b_{i+1}} \neq 1$ is in $\mathbf{F}[W]^{G}$. Therefore we may write $\alpha=\left(u_{i} u_{j}\right) / u_{i+1}=\prod_{k=1}^{m} u_{k}^{d_{i, j, k}}$ for some non-negative integers $d_{i, j, k}$. Note that $u_{r}$ does not divide $\alpha$ for all $r \leq i$ because $a_{r} \geq a_{i}>a_{i}+a_{j}-a_{i+1}$. Similarly $u_{r}$ does not divide $\alpha$ for all $r \geq j$ because $b_{r} \geq b_{j}>b_{i}+b_{j}-b_{i+1}$. Therefore $\alpha=\prod_{k=i+1}^{j-1} u_{k}^{d_{i, j, k}}$. For each pair $i, j$ with $1 \leq i, j \leq m$ and $j-i \geq 2$, fix such a factorization and define $R_{i, j}:=U_{i} U_{j}-U_{i+1} \prod_{k=i+1}^{j-1} U_{k}^{d_{i, j, k}} \in S$. Note that $R_{i, j} \in J$, that its lead term $\operatorname{LT}\left(R_{i, j}\right)=U_{i} U_{j}$ is quadratic, and that its other term has polynomial degree at least 2 .

Proposition 3.1. The $\left(\begin{array}{c}m-1 \\ 2\end{array}\right)$ elements

$$
\left\{R_{i, j} \mid 1 \leq i, j \leq m \text { and } j-i \geq 2\right\}
$$

form a Gröbner basis for $J$ and minimally generate $J$.

Proof. We assume, by way of contradiction, that the $R_{i, j}$ do not form a Gröbner basis for $J$. Choose a homogeneous element $f \in J$ with $\beta:=$ $\operatorname{LM}(f)$ minimal such that $\beta$ is not divisible by $\operatorname{LT}\left(R_{i, j}\right)$ for all $i, j$. We can assume that $f$ is monic, and, since $\mathbf{F}[W]^{G}$ is generated by monomials, that $f$ is a difference of two terms: $f=\prod_{k=1}^{m} U_{k}^{c_{k}}-\prod_{k=1}^{m} U_{k}^{d_{k}}$. Since $\mathrm{LT}\left(R_{i, j}\right)=U_{i} U_{j}$ for $1 \leq i, j \leq m, j-i \geq 2$ and no leading term divides $\beta$, we conclude that $\beta=U_{i}^{p} U_{i+1}^{q}$ for some $1 \leq i \leq m$, some positive integer $p$, and some non-negative integer $q$ (where $U_{m+1}:=1$ if $i=m$ ). Recalling that $\beta=\operatorname{LM}(f)$, we have $f=U_{i}^{p} U_{i+1}^{q}-\prod_{k=1}^{i+1} U_{k}^{d_{k}}$ for some non-negative integers $d_{k}$. Since neither $U_{i}$ nor $U_{i+1}$ can divide $f$ (by the minimality of $\beta$ ) we must have $f=U_{i}^{p} U_{i+1}^{q}-\prod_{k=1}^{i-1} U_{k}^{d_{k}}$. We will now show that such an $f$ cannot exist because $U_{i}^{p} U_{i+1}^{q}$ is the smallest monomial in $S$ that maps to $\pi\left(U_{i}^{p} U_{i+1}^{q}\right)=u_{i}^{p} u_{i+1}^{q}$. Applying $\pi$ to $f$, gives $0=$ 
$\pi\left(U_{i}^{p} U_{i+1}^{q}\right)-\pi\left(\prod_{k=1}^{i-1} U_{k}^{d_{k}}\right)=x^{p a_{i}+q a_{i+1}} y^{p b_{i}+q b_{i+1}}-x^{\sum_{k=1}^{i-1} d_{k} a_{k}} y^{\sum_{k=1}^{i-1} d_{k} b_{k}}$ and therefore $p a_{i}+q a_{i+1}=\sum_{k=1}^{i-1} d_{k} a_{k}$ and $p b_{i}+q b_{i+1}=\sum_{k=1}^{i-1} d_{k} b_{k}$. Therefore

$$
\frac{p b_{i}+q b_{i+1}}{p a_{i}+q a_{i+1}}=\frac{\sum_{k=1}^{i-1} d_{k} b_{k}}{\sum_{k=1}^{i-1} d_{k} a_{k}} .
$$

Now $p b_{i}+q b_{i+1} \geq(p+q) b_{i}$ and $p a_{i}+q a_{i+1} \leq(p+q) a_{i}$ and thus

$$
\frac{p b_{i}+q b_{i+1}}{p a_{i}+q a_{i+1}} \geq \frac{(p+q) b_{i}}{(p+q) a_{i}}=\frac{b_{i}}{a_{i}} .
$$

Similarly, $\sum_{k=1}^{i-1} d_{k} b_{k} \leq\left(\sum_{k=1}^{i-1} d_{k}\right) b_{i-1}$ and $\sum_{k=1}^{i-1} d_{k} a_{k} \geq\left(\sum_{k=1}^{i-1} d_{k}\right) a_{i-1}$ which implies that

$$
\frac{\sum_{k=1}^{i-1} d_{k} b_{k}}{\sum_{k=1}^{i-1} d_{k} a_{k}} \leq \frac{\left(\sum_{k=1}^{i-1} d_{k}\right) b_{i-1}}{\left(\sum_{k=1}^{i-1} d_{k}\right) a_{i-1}}=\frac{b_{i-1}}{a_{i-1}}
$$

Since $b_{i} / a_{i}>b_{i-1} / a_{i-1}$ we have a contradiction. Thus the elements $\operatorname{LT}\left(R_{i, j}\right)$ give a Gröbner basis for $J$ and so also generate $J$.

Finally, since the leading terms of the $R_{i, j}$ are distinct and quadratic, and their non-lead terms have polynomial degree greater than or equal to 2 , they minimally generate.

In Section 5 below, we will find the polynomial Betti numbers for a class of monomial ideals which includes the ideal LT $(J)$. Proposition 5.3 applied to $\operatorname{LT}(J)$ yields the following result:

Proposition 3.2. The polynomial Betti numbers for the ideal LT $(J)$ are:

$\beta_{i, j}= \begin{cases}1, & \text { for } i=-1, j=0 \\ (i+1)\left(\begin{array}{c}m \\ i+2\end{array}\right)-(m-1)\left(\begin{array}{c}m-2 \\ i\end{array}\right), & \text { for } i=0, \ldots, m-3, j=i+2 \\ 0, & \text { otherwise. }\end{cases}$

Since the non-zero polynomial Betti numbers $\beta_{i, j}$ for a fixed $i$ are concentrated in a single $j$, it follows that the minimal graded free resolution of $\operatorname{LT}(J)$ is pure. Lemma 2.1 then implies that the graded Betti numbers for the ideal $J$ are the same as for $\operatorname{LT}(J)$. Following the conventions relating Betti numbers of $J$ to Betti numbers of $S / J$, we obtain the following proposition.

Proposition 3.3. Let $W=W_{b} \oplus W_{c}$. Then the minimal free resolution of $\mathbf{F}[W]^{G} \cong S / J$ as an $S$-module is of the form

$$
0 \rightarrow M_{m-2} \stackrel{d_{m-2}}{\rightarrow} \cdots \rightarrow M_{2} \stackrel{d_{2}}{\rightarrow} M_{1} \stackrel{d_{1}}{\rightarrow} M_{0} \stackrel{d_{0}}{\rightarrow} \mathbf{F}[W]^{G} \rightarrow 0,
$$

where $\operatorname{rank}\left(M_{0}\right)=1$, and, for $1 \leq i \leq m-2, \operatorname{rank}\left(M_{i}\right)=i\left(\begin{array}{c}m \\ i+1\end{array}\right)-$ $(m-1)\left(\begin{array}{c}m-2 \\ i-1\end{array}\right)$. The basis element of $M_{0}$ is in polynomial degree 0 and 
the basis elements of $M_{i}$, for $1 \leq i \leq m-2$, have polynomial degree $i+1$.

Example 3.4. Let $G=\mathbf{Z} / 10$ and let $W=W_{b} \oplus W_{c}$ with $b=1$ and $c=2$. Then $\mathbf{F}[W]^{G}=\mathbf{F}[x, y]^{G}$ is generated by the monomials $u_{1}=x^{10}$, $u_{2}=x^{8} y, u_{3}=x^{6} y^{2}, u_{4}=x^{4} y^{3}, u_{5}=x^{2} y^{4}$, and $u_{6}=y^{5}$. The ideal of relations, $J$, has ten minimal generators:

$$
\begin{array}{lll}
R_{1,3}=U_{1} U_{3}-U_{2}^{2}, & R_{1,4}=U_{1} U_{4}-U_{2} U_{3}, & R_{1,5}=U_{1} U_{5}-U_{2} U_{4}, \\
R_{1,6}=U_{1} U_{6}-U_{2} U_{5}, & R_{2,4}=U_{2} U_{4}-U_{3}^{2}, & R_{2,5}=U_{2} U_{5}-U_{3} U_{4}, \\
R_{2,6}=U_{2} U_{6}-U_{3} U_{5}, & R_{3,5}=U_{3} U_{5}-U_{4}^{2}, & R_{3,6}=U_{3} U_{6}-U_{4} U_{5}, \\
R_{4,6}=U_{4} U_{6}-U_{5}^{2} . &
\end{array}
$$

The polynomial Betti numbers for $S / \operatorname{LT}(J)$ (see Example 5.4) are:

$$
\beta_{0,0}=1 \quad \beta_{1,2}=10 \quad \beta_{2,3}=20 \quad \beta_{3,4}=15 \quad \beta_{4,5}=4 \text {. }
$$

The graded Betti numbers for $S / \operatorname{LT}(J)$ and $S / J$ are:

\begin{tabular}{r|r|}
\hline$j:$ & 0 \\
\hline$\beta_{0, j}:$ & 1 \\
\hline
\end{tabular}

\begin{tabular}{|r|r|r|r|r|r|r|r|}
\hline$j:$ & 12 & 13 & 14 & 15 & 16 & 17 & 18 \\
\hline$\beta_{1, j}:$ & 1 & 1 & 2 & 2 & 2 & 1 & 1 \\
\hline
\end{tabular}

\begin{tabular}{|r|r|r|r|r|r|r|r|r|}
\hline$j:$ & 19 & 20 & 21 & 22 & 23 & 24 & 25 & 26 \\
\hline$\beta_{2, j}:$ & 1 & 2 & 3 & 4 & 4 & 3 & 2 & 1 \\
\hline
\end{tabular}

\begin{tabular}{|r|r|r|r|r|r|r|r|}
\hline$j:$ & 27 & 28 & 29 & 30 & 31 & 32 & 33 \\
\hline$\beta_{3, j}:$ & 1 & 2 & 3 & 3 & 3 & 2 & 1 \\
\hline
\end{tabular}

\begin{tabular}{|r|r|r|r|r|}
\hline$j:$ & 36 & 37 & 38 & 39 \\
\hline$\beta_{4, j}:$ & 1 & 1 & 1 & 1 \\
\hline
\end{tabular}

\section{Three Dimensional Case}

Now suppose $W=W_{b} \oplus W_{c} \oplus W_{d}$ is a 3 dimensional representation of $G$ with $0<b, c, d<n$ and $G \subset \operatorname{SL}(W)$. Then $\mathbf{F}[W]=\mathbf{F}[x, y, z]$ where $\sigma \cdot x=\epsilon^{b} x, \sigma \cdot y=\epsilon^{c} y$, and $\sigma \cdot z=\epsilon^{d} z$. Again we can choose a minimal generating set for the $\mathbf{F}$-algebra $\mathbf{F}[W]^{G}$ consisting of monomials. Since $0<b, c, d<n$ and $G \subset \operatorname{SL}(W)($ so that $b+c+d \equiv 0(\bmod n))$, the element $a=x y z$ is a minimal generator for $\mathbf{F}[W]^{G}$. The other generators can be chosen as follows:

$$
\text { - } u_{i}=x^{a_{i}} y^{b_{i}} \text { for } i=1,2, \ldots, r
$$

with $a_{1}>a_{2}>\cdots>a_{r} \neq 0$, and $0=b_{1}<b_{2}<\cdots<b_{r}$.

$$
\text { - } v_{j}=y^{c_{j}} z^{d_{j}} \text { for } j=1,2, \ldots, s
$$

with $c_{1}>c_{2}>\cdots>c_{s} \neq 0$, and $0=d_{1}<d_{2}<\cdots<d_{s}$.

$$
\text { - } w_{k}=z^{e_{k}} x^{f_{k}} \text { for } k=1,2, \ldots, t
$$

with $e_{1}>e_{2}>\cdots>e_{t} \neq 0$, and $0=f_{1}<f_{2}<\cdots<f_{t}$. 
Consider the short exact sequence

$$
0 \rightarrow J \rightarrow S=\mathbf{F}\left[A, B_{1}, B_{2}, \ldots, B_{r+s+t}\right] \stackrel{\pi}{\longrightarrow} \mathbf{F}[W]^{G} \rightarrow 0,
$$

where $A$ and the $B_{i}$ are indeterminants; $\pi(A)=a ; \pi\left(B_{i}\right)=u_{i}$, for $i=1,2, \ldots, r ; \pi\left(B_{r+j}\right)=v_{j}$, for $j=1,2, \ldots, s ; \pi\left(B_{r+s+k}\right)=w_{k}$, for $k=1,2, \ldots, t$; and $J$ is the ideal of relations. We set $\operatorname{deg}(A)=$ $\operatorname{deg}(a)=3 ; \operatorname{deg}\left(B_{i}\right)=\operatorname{deg}\left(\pi\left(B_{i}\right)\right)$, for $i=1, \ldots, r+s+t$ and work with the following monomial order on $S$, for monomials

$$
\begin{aligned}
\alpha & =A^{g_{0}} B_{1}^{g_{1}} B_{2}^{g_{2}} \cdots B_{r+s}^{g_{r+s}} B_{r+s+t}^{g_{r+s+1}} B_{r+s+t-1}^{g_{r+s+2}} \cdots B_{r+s+1}^{g_{r+s+t}} \text { and } \\
\beta & =A^{h_{0}} B_{1}^{h_{1}} B_{2}^{h_{2}} \cdots B_{r+s}^{h_{r+s}} B_{r+s+t}^{h_{r+s+1}} B_{r+s+t-1}^{h_{r+s+2}} \cdots B_{r+s+1}^{h_{r+s+t}},
\end{aligned}
$$

we say $\alpha<\beta$ if

(1) $\operatorname{deg}(\alpha)<\operatorname{deg}(\beta)$,

(2) $\operatorname{deg}(\alpha)=\operatorname{deg}(\beta)$ and $g_{0}>h_{0}$,

(3) $\operatorname{deg}(\alpha)=\operatorname{deg}(\beta), g_{0}=h_{0}$, and $g_{i}<h_{i}$ for the largest $i$ with $g_{i} \neq h_{i}$.

(The reader should note the unusual order of the variables in the above expansions of $\alpha$ and $\beta$.)

Note that the three ordered sets $T_{1}=\left\{B_{1}, B_{2}, \ldots, B_{r+1}\right\}, T_{2}=$ $\left\{B_{r+1}, B_{r+2}, \ldots, B_{r+s+1}\right\}$, and $T_{3}=\left\{B_{1}, B_{r+s+t}, B_{r+s+t-1}, \ldots, B_{r+s+1}\right\}$ map to the monomials in $\mathbf{F}[W]^{G}$ not divisible by $z$, by $x$, and by $y$, respectively. This monomial order is carefully chosen to be compatible with these three sets and to ensure that the elements of the minimal generating set given in Proposition 4.2 below form a Gröbner basis for $J$.

For the remainder of this section, we will let $m=r+s+t$.

Now we construct a minimal generating set for $J$. The following distance formula will be useful.

Definition 4.1. For $1 \leq i, j \leq m$ define $\|i-j\|:=\min \{|i-j|, m-$ $|i-j|\}$.

This is just the natural distance function on the vertices of a labelled m-gon.

Consider the products $B_{i} B_{j}$ with $1 \leq i<j \leq m$ and $\|i-j\| \geq 2$. If $B_{i}$ and $B_{j}$ are both in $T_{1}$ (they map to monomials not divisible by $z$ ), or both in $T_{2}$, or both in $T_{3}$, then construct $R_{i, j}$, with leading term $B_{i} B_{j}$, as in the two dimensional case. If $B_{i}$ and $B_{j}$ are not both in the same $T_{k}$, then $\pi\left(B_{i} B_{j}\right)$ is divisible by $a=x y z$, so $\pi\left(B_{i} B_{j}\right) / a$ is in $\mathbf{F}[W]^{G}$. Choose a monomial $\alpha_{i, j} \in S$ with $\pi\left(\alpha_{i, j}\right)=\pi\left(B_{i} B_{j}\right) / a$ and define $R_{i, j}=B_{i} B_{j}-A \alpha_{i, j}$, which has leading term $B_{i} B_{j}$ (since a monomial divisible by $A$ is smaller than another of the same degree 
which is not divisible by $A$ ). Of course, for all $i, j$, the non-lead term of $R_{i, j}$ has polynomial degree greater than or equal to 2 .

Proposition 4.2. The $\frac{m(m-3)}{2}$ elements

$$
\left\{R_{i, j} \mid 1 \leq i<j \leq m \text { and }\|i-j\| \geq 2\right\}
$$

form a Gröbner basis for $J$ and minimally generate $J$.

Proof. Note that since $\pi$ preserves degree, the ideal $J$ is homogeneous, so can be (minimally) generated by homogeneous elements. Also note that if $f$ is any homogeneous element of $S$ for which $A$ divides $\operatorname{LM}(f)$ then $A$ divides $f$. From this, it follows that $A$ cannot divide the lead monomial of any minimal homogeneous generator of $J$.

We assume, by way of contradiction, that the $R_{i, j}$ do not form a Gröbner basis for $J$. Choose a homogeneous element $f \in J$ with $\beta:=\operatorname{LM}(f)$ minimal such that $\beta$ is not divisible by $\operatorname{LT}\left(R_{i, j}\right)$ for all $i, j$. We can assume that $f$ is monic, and, since $\mathbf{F}[W]^{G}$ is generated by monomials, that $f$ is a difference of two terms: $f=\prod_{k=1}^{m} B_{k}^{g_{k}}-A^{h_{0}} \prod_{k=1}^{m} B_{k}^{h_{k}}$. Since $\operatorname{LT}\left(R_{i, j}\right)=B_{i} B_{j}$ (for $\|i-j\| \geq 2$ ) and no leading term divides $\beta$, we conclude that $\beta=B_{i}^{p} B_{i+1}^{q}$ for some $1 \leq i \leq m$, some positive integer $p$, and some non-negative integer $q$ (where $B_{m+1}:=B_{1}$ if $i=m)$. But these possible lead terms involve at most two variables from $\mathbf{F}[W]$, so are the minimal monomials mapping to $\pi(\beta)$ in $\mathbf{F}[W]^{G}$ as in the two dimensional case. Hence these monomials cannot be lead terms for relations and so the $R_{i, j}$ form a Gröbner basis for $J$ and so also generate $J$.

Since the leading terms of the $R_{i, j}$ are distinct and quadratic, and their non-lead terms have polynomial degree greater than or equal to 2 , they minimally generate.

We now describe the minimal graded free resolution of $\mathbf{F}[W]^{G} \cong S / J$.

The following result allows us to work modulo the principle ideal generated by the element $A \in S$.

Lemma 4.3. ([CHW] Lemma 5.3) Let I be a homogeneous ideal in the graded ring $R=\mathbf{F}\left[x_{1}, \ldots, x_{m}\right]$ and put $Q=R / I$. Suppose $r \in R$ which is not a zero divisor for $R$ or for $Q$. Let $\left(M_{*}, d_{*}\right)$ be a minimal graded free resolution of $Q$ as an $R$-module. Then the complex $\left(M_{*} /(r), d_{*} /(r)\right)$ is a minimal graded free resolution of $Q /(r)$ as an $R /(r)$-module. In particular, the graded Betti numbers of these resolutions are the same.

The element $A \in S=\mathbf{F}\left[A, B_{1}, \ldots, B_{m}\right]$ is not a zero divisor for $S$ or $S / J$ so the above Lemma applies. Let $\bar{S}=S /(A)$ and $\bar{J}=J /(A)$, 
then the lemma implies that $S / J$ (which is isomorphic to $\mathbf{F}[W]^{G}$ ) and $\bar{S} / \bar{J}$ have the same graded Betti numbers.

In Section 5, we will find the polynomial Betti numbers for the lead term ideal $\operatorname{LT}(\bar{J})$, which is the monomial ideal in $\bar{S}=\mathbf{F}\left[B_{1}, \ldots, B_{m}\right]$ generated by $\left\{B_{i} B_{j} \mid 1 \leq i<j \leq m\right.$ and $\left.\|i-j\| \geq 2\right\}$. Proposition 5.3 applied to $\operatorname{LT}(\bar{J})$ yields the following result:

Proposition 4.4. The polynomial Betti numbers for the ideal $\operatorname{LT}(\bar{J})$ are:

$$
\beta_{i, j}= \begin{cases}1, & \text { for } i=-1 \text { and } j=0 \\
(i+1)\left(\begin{array}{c}
m \\
i+2
\end{array}\right)-m\left(\begin{array}{c}
m-2 \\
i
\end{array}\right), & \text { for } i=0, \ldots, m-4 \text { and } j=i+2 \\
1, & \text { for } i=m-3 \text { and } j=m ; \\
0, & \text { otherwise. }\end{cases}
$$

Since the non-zero polynomial Betti numbers $\beta_{i, j}$ for a fixed $i$ are concentrated in a single $j$, it follows that the minimal graded free resolution of $\operatorname{LT}(\bar{J})$ is pure, so Lemma 2.1 implies that the graded Betti numbers for the ideal $\bar{J}$ (which are also the same as for $J$ ) are the same as for $\operatorname{LT}(\bar{J})$. Again, following the conventions relating Betti numbers of $J$ to Betti numbers of $S / J$, gives the following proposition.

Proposition 4.5. Let $W=W_{b} \oplus W_{c} \oplus W_{d}$ with $0<b, c, d<n$ and $b+c+d \equiv 0(\bmod n)$. Then the minimal graded free resolution of $\mathbf{F}[W]^{G} \cong S / J$ as an $S$-module is of the form

$$
0 \rightarrow M_{m-2} \stackrel{d_{m-2}}{\rightarrow} \cdots \rightarrow M_{2} \stackrel{d_{2}}{\rightarrow} M_{1} \stackrel{d_{1}}{\rightarrow} M_{0} \stackrel{d_{0}}{\rightarrow} \mathbf{F}[W]^{G} \rightarrow 0
$$

where $\operatorname{rank}\left(M_{0}\right)=\operatorname{rank}\left(M_{m-2}\right)=1$, and, for $1 \leq i \leq m-3, \operatorname{rank}\left(M_{i}\right)=$ $i\left(\begin{array}{c}m \\ i+1\end{array}\right)-m\left(\begin{array}{c}m-2 \\ i-1\end{array}\right)$. The basis element of $M_{0}$ is in polynomial degree 0 ; the basis elements of $M_{i}, 1 \leq i \leq m-3$, have polynomial degree $i+1$; and the basis element of $M_{m-2}$ has polynomial degree $m$.

Remark 4.6. A special case of the above result, when $n=1+e+e^{2}$, $b=1, c=e$, and $d=e^{2}$, can be found in [CHW]. In that paper, we explicitly described basis elements and differentials for the resolution of $\bar{S} / \operatorname{LT}(\bar{J})$ and then constructed a contracting homotopy to prove exactness. The techniques used in Section 5 are much simpler.

Example 4.7. Let $G=\mathbf{Z} / 6$ and let $W=W_{b} \oplus W_{c} \oplus W_{d}$ with $b=1$, $c=2$, and $d=3$. Then $\mathbf{F}[W]^{G}=\mathbf{F}[x, y, z]^{G}$ is generated by the monomials $a=x y z, b_{1}=x^{6}, b_{2}=x^{4} y, b_{3}=x^{2} y^{2}, b_{4}=y^{3}, b_{5}=z^{2}$, and $b_{6}=x^{3} z$. The ideal of relations, $J$, has nine minimal generators: 


$$
\begin{array}{lll}
R_{1,3}=B_{1} B_{3}-B_{2}^{2}, & R_{1,4}=B_{1} B_{4}-B_{2} B_{3}, & R_{1,5}=B_{1} B_{5}-B_{6}^{2} \\
R_{2,4}=B_{2} B_{4}-B_{3}^{2}, & R_{2,5}=B_{2} B_{5}-A B_{6}, & R_{2,6}=B_{2} B_{6}-A B_{1} \\
R_{3,5}=B_{3} B_{5}-A^{2}, & R_{3,6}=B_{3} B_{6}-A B_{2}, & R_{4,6}=B_{4} B_{6}-A B_{3} .
\end{array}
$$

The polynomial Betti numbers for $S / \operatorname{LT}(J)$ (see Example 5.4) are:

$$
\beta_{0,0}=1 \quad \beta_{1,2}=9 \quad \beta_{2,3}=16 \quad \beta_{3,4}=9 \quad \beta_{4,6}=1 .
$$

The graded Betti numbers for $S / \operatorname{LT}(J)$ and $S / J$ are:

\begin{tabular}{|r||r|r|r|r|r|r|r|r|r|r|r|r|r|r|r|r|r|}
\hline$j:$ & 0 & $\ldots$ & 6 & 7 & 8 & 9 & 10 & 11 & 12 & 13 & 14 & 15 & 16 & 17 & 18 & $\ldots$ & 24 \\
\hline \hline$\beta_{0, j}:$ & 1 & & & & & & & & & & & & & & & & \\
\hline$\beta_{1, j}:$ & & & 1 & 2 & 3 & 2 & 1 & & & & & & & & & & \\
\hline$\beta_{2, j}:$ & & & & & & & 2 & 4 & 4 & 4 & 2 & & & & & & \\
\hline$\beta_{3, j}:$ & & & & & & & & & & & 1 & 2 & 3 & 2 & 1 & & \\
\hline$\beta_{4, j}:$ & & & & & & & & & & & & & & & & & 1 \\
\hline
\end{tabular}

\section{Graded Betti Numbers for Some Monomial Ideals}

Let $R=\mathbf{F}\left[x_{1}, \ldots, x_{m}\right]$, and let $I$ be an ideal in $R$ that is generated by square-free quadratic monomials. Then $I$ can be described as the edge ideal of a simple graph. Here are the relevant definitions and results we will need (we follow the exposition of [HvT]).

Let $X$ be a finite graph with vertex set $V_{X}=\left\{x_{1}, x_{2}, \ldots, x_{n}\right\}$ and edge set $E_{X}$. The graph $X$ is called simple if $X$ has no loops or multiple edges. A simple graph need not be connected - we let $\# \operatorname{comp}(X)$ denote the number of components of $X$. When $Y$ is a subset of $V_{X}$, the induced subgraph of $X$ on the vertex set $Y$, denoted $X_{Y}$, is the subgraph of $X$ with vertices $Y$ and edge set consisting of the edges in $X$ connecting vertices in $Y$. The complement of a graph $X$, denoted $X^{c}$, is the graph whose vertex set is the same as $X$, but whose edge set is the complement of the edge set of $X$ : the edge $\left\{x_{i}, x_{j}\right\} \in E_{X^{c}}$ if and only if $\left\{x_{i}, x_{j}\right\} \notin E_{X}$. The complete graph on a set of vertices is the graph with exactly one edge for each pair of distinct vertices.

A simplicial complex $\Delta$ on a vertex set $V_{\Delta}$ is a collection of subsets of $V_{\Delta}$ such that: (i) for each vertex $x_{i} \in V_{\Delta}$, the set $\left\{x_{i}\right\} \in \Delta$, and (ii) for each set $F$ in $\Delta$, if $E \subseteq F$, then $E \in \Delta$. An element $F$ of a simplicial complex $\Delta$ is also called a face of $\Delta$. The dimension of a face $F$ of $\Delta$, denoted $\operatorname{dim} F$, is defined to be $|F|-1$, where $|F|$ denotes the number of vertices in $F$. The maximal faces of $\Delta$ under inclusion are called the facets of $\Delta$. There is a one-to-one correspondence between simplicial complexes and their facet sets.

If $\Delta$ is a simplicial complex with vertex set $V_{\Delta}=\left\{x_{1}, \ldots, x_{m}\right\}$, then we can associate two ideals to $\Delta$ in the polynomial ring $R=$ $\mathbf{F}\left[x_{1}, \ldots, x_{m}\right]$. (By abuse of notation we use $x_{i}$ to denote both a vertex 
of $\Delta$ and a variable in $R$.) For a face $F$ of $\Delta$, we let $x^{F}$ be the monomial $\prod_{x \in F} x$ in $R$. The facet ideal, $\mathcal{I}(\Delta)$, and Stanley-Reisner ideal, $I_{\Delta}$, of $\Delta$ are defined as follows:

$\mathcal{I}(\Delta)=\left\langle x^{F}\right| F$ is a facet of $\left.\Delta\right\rangle$ and $I_{\Delta}=\left\langle x^{F}\right| F \subseteq V_{\Delta}$ and $\left.F \notin \Delta\right\rangle$.

Note that a connected graph $X$ can be thought of as a simplicial complex with facet set equaling its edge set. In this situation, the facet ideal $\mathcal{I}(X)$ is also called the edge ideal. Additionally, we can associate another simplicial complex to $X$ : the clique complex of $X$ is the simplicial complex $\Delta(X)$ where $F=\left\{x_{i_{1}}, \ldots, x_{i_{j}}\right\} \in \Delta(X)$ if and only if the induced subgraph $X_{F}$ is a complete graph. Note that when $X$ is a simple graph, then the edge ideal $\mathcal{I}(X)$ is generated by square-free quadratic monomials and is equal to the Stanley-Reisner ideal of the clique complex of its complement, that is, $\mathcal{I}(X)=I_{\Delta\left(X^{c}\right)}$. The following result uses Hochster's and Eagon-Reiner's formulas to compute the graded Betti numbers of $\mathcal{I}(X)$.

Theorem 5.1. ([HvT], Theorem 3.2.1) Let $X$ be a simple graph with edge ideal $\mathcal{I}(X)$. Then

$$
\beta_{i, j}(\mathcal{I}(X))=\sum_{Y \subseteq V_{X},|Y|=j} \operatorname{dim}_{\mathbf{F}} \widetilde{H}_{j-i-2}\left(\Delta\left(X_{Y}^{c}\right), \mathbf{F}\right) \text { for all } i, j \geq 0
$$

In the case when $j=i+2$, the above formula involves only the 0 -th homology, so we also have:

Theorem 5.2. ([HvT], Theorem 3.2.4) Let $X$ be a simple graph with edge ideal $\mathcal{I}(X)$. Then for all $i \geq 0$,

$$
\beta_{i, i+2}(\mathcal{I}(X))=\sum_{Y \subseteq V_{X},|Y|=i+2}\left(\# \operatorname{comp}\left(X_{Y}^{c}\right)-1\right) .
$$

We now apply these results to compute the Betti numbers for $\mathcal{I}(X)$ for a specific collection of graphs $X$.

Let $X[0]$ be the complete graph with vertices $V_{X}=\left\{x_{1}, \ldots, x_{m}\right\}$ and all $\left(\begin{array}{c}m \\ 2\end{array}\right)$ edges. The $m$ edges $\left\{\left\{x_{i}, x_{i+1}\right\} \mid 1 \leq i \leq m\right\}$ (where $x_{m+1}$ will mean $x_{1}$ ) will be called adjacent edges. Let $X[s]$ be some graph obtained from the complete graph $X[0]$ by deleting $s$ of the adjacent edges where $1 \leq s \leq m$.

Proposition 5.3. For $s=0, \ldots, m-1$, the graded Betti numbers of $\mathcal{I}(X[s])$ are:

$$
\beta_{i, j}= \begin{cases}1, & \text { for } i=-1 \text { and } j=0 \\
(i+1)\left(\begin{array}{c}
m \\
i+2
\end{array}\right)-s\left(\begin{array}{c}
m-2 \\
i
\end{array}\right), & \text { for } i=0, \ldots, m-2 \text { and } j=i+2 \\
0, & \text { otherwise. }\end{cases}
$$


For $s=m$, the graded Betti numbers of $\mathcal{I}(X[m])$ are:

$$
\beta_{i, j}= \begin{cases}1, & \text { for } i=-1 \text { and } j=0 \\
(i+1)\left(\begin{array}{c}
m \\
i+2
\end{array}\right)-m\left(\begin{array}{c}
m-2 \\
i
\end{array}\right), & \text { for } i=0, \ldots, m-4 \text { and } j=i+2 \\
1, & \text { for } i=m-3 \text { and } j=m \\
0, & \text { otherwise. }\end{cases}
$$

Proof. By definition, $\beta_{-1,0}=1$ and $\beta_{-1, j}=0$ if $j \neq 0$.

For $i \geq 0$, we use Theorem 5.1 and Theorem 5.2. It is easy to see that $X[m]^{c}$ is just an $m$-cycle. It is also easy to see that $X[s]^{c}$ is a subgraph of this $m$-cycle with the same vertices but only $s$ edges. Hence for any subset $Y \subseteq V_{X}$, each component of the clique complex $\Delta\left(X[s]_{Y}^{c}\right)$ is simply connected unless $s=m=|Y|$.

First consider the case where $j=|Y|<m$ so that

$$
\widetilde{H}_{j-i-2}\left(\Delta\left(X[s]_{Y}^{c}\right), \mathbf{F}\right)=0
$$

unless $j-i-2=0$, and consequently, $\beta_{i, j}=0$ unless $j=i+2$. We use Theorem 5.2 to compute the remaining Betti numbers for $j=i+2$. Since any acyclic graph with $j$ vertices and $t$ edges has $j-t$ components, we see that

$$
\begin{aligned}
\beta_{j-2, j}(\mathcal{I}(X[s])) & =\sum_{Y \subseteq V_{X},|Y|=j}\left(\# \operatorname{comp}\left(X[s]_{Y}^{c}\right)-1\right) \\
& =\sum_{Y \subseteq V_{X},|Y|=j} j-1-\# E_{X[s]_{Y}^{c}} \\
& =(j-1)\left(\begin{array}{c}
m \\
j
\end{array}\right)-\sum_{Y \subseteq V_{X},|Y|=j} \# E_{X[s]_{Y}^{c}} .
\end{aligned}
$$

Since each of the $s$ edges of $X[s]^{c}$ lies in exactly $\left(\begin{array}{c}m-2 \\ j-2\end{array}\right)$ subsets $Y \subseteq V_{X}$ of size $j$ we see that

$\beta_{j-2, j}(\mathcal{I}(X[s]))=(j-1)\left(\begin{array}{c}m \\ j\end{array}\right)-s\left(\begin{array}{c}m-2 \\ j-2\end{array}\right)=(i+1)\left(\begin{array}{c}m \\ i+2\end{array}\right)-s\left(\begin{array}{c}m-2 \\ i\end{array}\right)$

for $i=j-2$ as required.

Finally, we consider the case where $j=|Y|=m$ (so necessarily, $s=$ $m$ and $\left.Y=V_{X}\right)$ and $i \geq 0$. By Theorem 5.1 we have $\beta_{i, m}(\mathcal{I}(X[m]))=$ $\operatorname{dim}_{\mathbf{F}} \widetilde{H}_{m-i-2}(\Gamma, \mathbf{F})$, where $\Gamma=\Delta\left(X[m]_{Y}^{c}\right)=\Delta\left(X[m]^{c}\right)$ is the clique complex of the $m$-cycle, i.e., $\Gamma$ is a circle. Thus $\beta_{m-3, m}(\mathcal{I}(X[m]))=1$ and $\beta_{i, m}(\mathcal{I}(X[m]))=0$ for $i \neq m-3$.

Note that the lead term ideals in Sections 3 and 4 correspond to the edge ideals $\mathcal{I}(X[m-1])$ and $\mathcal{I}(X[m])$, respectively. Also note that the 
resolutions for $\mathcal{I}(X[s]), s=0, \ldots, m-1$ are linear (see [RvT] for the definition of a linear resolution).

Example 5.4. Here are the polynomial Betti numbers, $\beta_{i, j}$, for the $\mathcal{I}(X[s])$ when $m=6$. The first column corresponds to $j=0$, the last non-zero entry (for $\beta_{3}(\mathcal{I}(X[6])$ ) corresponds to $j=6$, and all other entries correspond to $j=i+2$.

\begin{tabular}{|c||r|r|r|r|r|r|}
\hline$i=$ & -1 & 0 & 1 & 2 & 3 & 4 \\
\hline \hline$\beta_{i}(\mathcal{I}(X[0]))$ & 1 & 15 & 40 & 45 & 24 & 5 \\
\hline$\beta_{i}(\mathcal{I}(X[1]))$ & 1 & 14 & 36 & 39 & 20 & 4 \\
\hline$\beta_{i}(\mathcal{I}(X[2]))$ & 1 & 13 & 32 & 33 & 16 & 3 \\
\hline$\beta_{i}(\mathcal{I}(X[3]))$ & 1 & 12 & 28 & 27 & 4 & 2 \\
\hline$\beta_{i}(\mathcal{I}(X[4]))$ & 1 & 11 & 24 & 21 & 8 & 1 \\
\hline$\beta_{i}(\mathcal{I}(X[5]))$ & 1 & 10 & 20 & 15 & 4 & 0 \\
\hline$\beta_{i}(\mathcal{I}(X[6]))$ & 1 & 9 & 16 & 9 & 1 & 0 \\
\hline
\end{tabular}

Remark 5.5. One might notice that the final row of this table is symmetric. In the application to rings of invariants in Section 4, this corresponds to the fact that the invariant ring $\mathbf{F}[W]^{G}$ is Gorenstein when $G \subseteq \mathrm{SL}(W)$ (see Wat]) so its minimal graded free resolution is self-dual.

Remarks 5.6. Minimal resolutions for $\mathcal{I}(X[0])$ and $\mathcal{I}(X[m])$ were described in [CHW], where explicit basis elements and differentials were given and the complex was proved to be exact using a contracting homotopy. Also, the Betti numbers for $\mathcal{I}(X[0])$ were found in [J], Section 5, and in [RvT], Example 2.10. The Betti numbers for $\mathcal{I}(X[s])$, for $s=0, m-1$, and $m$, go back even further to a paper on rational singularities by Wahl Wah.

\section{REFERENCES}

[CHW] H.E.A. Campbell, J.C. Harris and David L. Wehlau, Internal Duality for Resolutions of Rings, J. of Algebra 215 (1999), 1-33.

[CLO] D. Cox, J. Little, and D. O'Shea, Ideals, varieties, and algorithms, SpringerVerlag, 1992.

[HvT] H. T. Ha and A. Van Tuyl, Resolutions of Square-Free Monomial Ideals Via Facet Ideals: A Survey, arXiv:math/0604301v2 [Math.AC] 28 May 2007.

[J] S. Jacque, Betti Numbers of Graph Ideals, arXiv:math.AC/0410107 v1, 5 October 2004.

[M-S] E. Miller and B. Sturmfels, Combinatorial Commutative Algebra. Springer GTM 227, Springer, 2004.

[RvT] M. Roth and A. Van Tuyl, On the Linear Strand of an Edge Ideal, arXiv:math.AC/0411181 v2, 12 April 2006. 
[Wah] J. M. Wahl, Equations defining rational singularities, Ann. Sci. École Norm. Sup. 10 (1977), 231-264.

[Wat] K. Watanabe, Certain invariant subrings are Gorenstein. I, Osaka J. Math. 11, No. 1 (1974), 1-8.

Department of Mathematics and Natural Sciences, D'Youville College, Buffalo, NY, USA, 14201

E-mail address: harrisj@dyc.edu

Department of Mathematics and Computer Science, Royal Military College, Kingston, Ontario, Canada, K7K 5L0

E-mail address: wehlau@rmc.ca 